\title{
ON APPROACH TO ANALYZE NONLINEAR MODEL OF MASS AND HEAT TRANSPORT DURING GAS PHASE EP- ITAXY. A POSSIBILITY TO IMPROVE PROPERTIES OF FILMS
}

\author{
E.L. Pankratov, E.A. Bulaeva \\ Nizhny Novgorod State University, 23 Gagarin avenue, \\ Nizhny Novgorod, 603950, \\ Russia
}

\section{ABSTRACT}

In this paper we introduce an analytical approach to calculate the distribution of velocity of flow of the gas mixture, the concentration distributions of the growth component and temperature fields in the zone of deposition of semiconductor layers in chemical vapor deposition using a reaction chamber with a rotating disk substrate holder. The results of analysis of the temperature and concentration fields in the reduced and atmospheric conditions ( $0.1 \mathrm{~atm}$.) pressure in the reaction chamber obtained based on the proposed theoretical models.

\section{KEYWORDS}

Gas phase epitaxy; semiconductor layer; convection diffusion model.

\section{INTRODUCTION}

At the present time one of main technological processes of manufacturing of devices of microand nanoelectronics is manufacturing semiconductor layers by epitaxy from gas phase and molecular beams [1-5]. Methods of grown are intensively improving. At the same time modeling of physical processes that occur during the growth of epitaxial layers is weakly elaborated. In this situation relations between technological parameters, which determine kinetic and mechanisms of growth of epitaxial layers, could leads to essential errors. Main aim of the present paper is development of methods of modeling of several physical processes, which are existing during epitaxy from gas phase in a reaction chamber with rotating disk keeper of substrate (see Fig. 1).

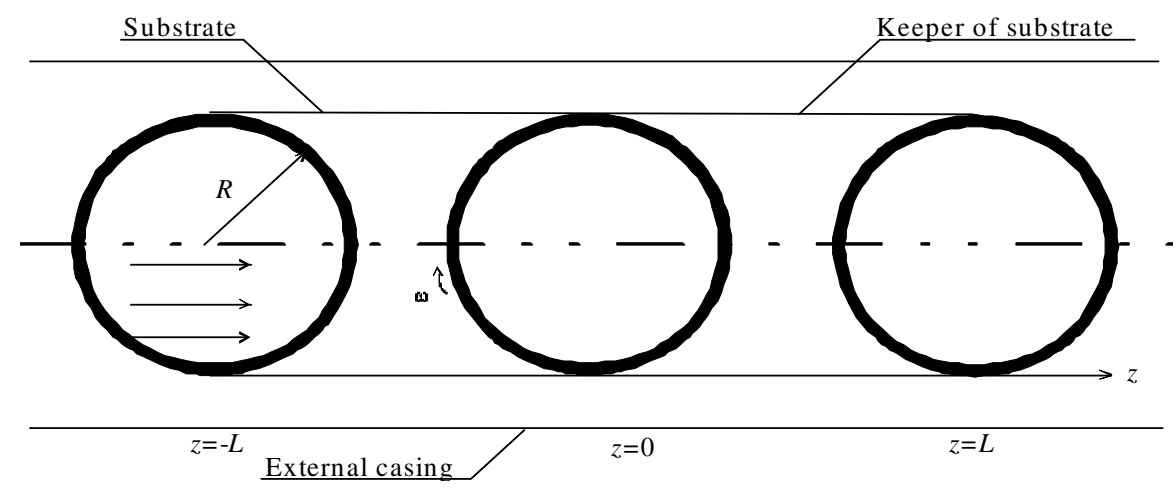

Fig. 1. Schematic representation of reaction chamber for gas phase epitaxy 
Similar technological scheme is main scheme for epitaxy from gas phase with using organo- metallic compounds (MOC) and hydrides as initial materials (MOCVD) framework one of perspective approaches of manufacturing of semiconductor nanoheterostructures [2,3]. The considered in Fig. 1 reactor includes into itself high-frequency heater of graphite keeper of substrate. A flow of mixture of gases coming into the considered reaction chamber. Several components of the mixture of gases, which chemically reacting in the considered chamber during growth of an epitaxial layer. Another component of the considered mixture of gases is hydrogen, which is considered as gas-carrier.

\section{Method of Solution}

To model physical processes, which are existing during growth epitaxial growth, we assume, that vector of linear speed of vector linear velocity entering the reaction chamber the flow perpendicular to the surface of the substrate holder disc keeper of substrate, which rotates with angular velocity $\omega$. In this paper we analyzed flow of the mixture of gases at atmosphere pressure and at low pressure ( $\sim, 1$ of atmosphere pressure) in reaction chamber, distribution of temperature and distribution of concentration of growth component in growth zone of epitaxial layer.

We determine required values by solving the second Fick's and Fourier laws for convective diffusion and the Navier-Stokes equation. We calculate distribution of considered concentration at the distance from the surface of disk of keeper of substrate with radius $R$, which approximately equal to thickness of diffusion layer [3]. We determine spatio-temporal distribution of temperature by solution the following heat equation [6]

$$
c(T) \frac{\partial T}{\partial t}=p+\operatorname{div}[\lambda \cdot \operatorname{grad}(T)-\mathbf{v} \cdot c(T) \cdot T \cdot C],
$$

where $v$ is the speed of mixture of gases; $c(T)$ is the heat capacitance; $T(r, \varphi, z, t)$ is the spatiotemporal distribution of temperature; $p$ is the density of power, which exudes in the system keeper of substrate - substrate; $C(r, \varphi, z, t)$ is the spatio-temporal distribution of concentration of growth component, which was generated during chemical reaction.

During solution of the Eq.(1) it is necessary to take into account flowing of gas and appropriate distribution of concentration of growth component. We determine the required values by joint solution of the Navier-Stokes equation and the second Fourier law with convective term. The equations could be written as

$$
\begin{gathered}
\frac{\partial v}{\partial t}+(v \cdot \nabla) v=-\nabla\left(\frac{P}{\rho}\right)+v \Delta v, \\
\frac{\partial C}{\partial t}=\operatorname{div}[D \cdot \operatorname{grad} C-v \cdot C] .
\end{gathered}
$$

Here $D$ is the diffusion component of growth component; $P$ is the pressure; $\rho$ is the density; $v$ is the kinematic viscosity. We consider regime of limiting flow of diffusion mass transport of growth component. In this limiting case we assume, that all particles of growth component precipitating and ordering on the substrate. We also assume, that flow is homogenous and one dimension in the inlet of reaction zone. In this situation boundary and initial conditions could be written as 
International Journal on Organic Electronics (IJOE) Vol.6, No.4, October 2017.

$$
\begin{gathered}
C(r, \varphi,-L, t)=C_{0}, C(r, \varphi, 0, t)=0, C(r, 0, z, t)=C(r, 2 \pi, z, t), C(r, \varphi, z, 0)=C_{0} \delta(z+L), C(0, \varphi, z, t) \neq \infty, \\
\left.\frac{\partial C(r, \varphi, z, t)}{\partial \varphi}\right|_{\varphi=0}=\left.\frac{\partial C(r, \varphi, z, t)}{\partial \varphi}\right|_{\varphi=2 \pi},\left.\frac{\partial C(r, \varphi, z, t)}{\partial r}\right|_{r=R}=0,-\left.\lambda \frac{\partial T(r, \varphi, z, t)}{\partial r}\right|_{r=R}=\sigma T^{4}(R, \varphi, z, t), \\
\left.\frac{\partial T(r, \varphi, z, t)}{\partial \varphi}\right|_{\varphi=0}=\left.\frac{\partial T(r, \varphi, z, t)}{\partial \varphi}\right|_{\varphi=2 \pi},-\left.\lambda \frac{\partial T(r, \varphi, z, t)}{\partial z}\right|_{z=-L}=\sigma T^{4}(r, \varphi,-L, t), T(r, 0, z, t)= \\
\begin{array}{c}
T(r, 2 \pi, z, t), T(r, \varphi, z, 0)=T_{r},\left.\frac{\partial v_{r}(r, \varphi, z, t)}{\partial r}\right|_{r=0}=\left.\frac{\partial v_{r}(r, \varphi, z, t)}{\partial r}\right|_{r=R},\left.\frac{\partial v_{\varphi}(r, \varphi, z, t)}{\partial \varphi}\right|_{\varphi=0}=\left.\frac{\partial v_{\varphi}(r, \varphi, z, t)}{\partial \varphi}\right|_{\varphi=2 \pi}, \\
\left.\frac{\partial v_{r}(r, \varphi, z, t)}{\partial r}\right|_{r=0}=\left.\frac{\partial v_{r}(r, \varphi, z, t)}{\partial r}\right|_{r=R},\left.\frac{\partial v_{\varphi}(r, \varphi, z, t)}{\partial \varphi}\right|_{\varphi=0}=\left.\frac{\partial v_{\varphi}(r, \varphi, z, t)}{\partial \varphi}\right|_{\varphi=2 \pi}, T(0, \varphi, z, t) \neq \infty,(4) \\
-\left.\lambda \frac{\partial T(r, \varphi, z, t)}{\partial z}\right|_{z=L}=\sigma T^{4}(r, \varphi, z, t), v_{r}(r, \varphi,-L, t)=0, v_{r}(r, \varphi, 0, t)=0, v_{r}(r, \varphi, L, t)=0, T(r, \varphi, z, 0) \\
=T_{r}, v_{r}(r, 0, z, t)=v_{r}(r, 2 \pi, z, t), v_{r}(0, \varphi, z, t) \neq \infty, v_{\varphi}(r, \varphi, 0, t)=\omega r, v_{\varphi}(r, \varphi,-L, t)=0, v_{\varphi}(r, \varphi, L, t)=0, \\
v_{\varphi}(r, 0, z, t)=v_{\varphi}(r, 2 \pi, z, t), v_{\varphi}(0, \varphi, z, t) \neq \infty, v_{z}(r, \varphi,-L, t)=V_{0}, v_{z}(r, \varphi, 0, t)=V_{0}, v_{z}(r, \varphi, L, t)=0,
\end{array} \\
v_{z}(r, 0, z, t)=v_{z}(r, 2 \pi, z, t), v_{z}(0, \varphi, z, t) \neq \infty, v_{r}(r, \varphi, z, 0)=0, v_{\varphi}(r, \varphi, z, 0)=0, v_{z}(r, \varphi,-L, 0)=V_{0},
\end{gathered}
$$

Here $\sigma$ is the Stefan-Boltzmann constant; $T_{r}$ is the temperature of cooled by water walls of reaction chamber; $v_{0}$ is the linear speed of gas flow in inlet area of growth zone; $r, \varphi, z$ and $t$ are the cylindrical coordinates and time; $C_{0}$ is the concentration of growth component in flow of gas mixture in inlet area of area of growth of layers; $S$ is the surface of keeper of substrate with substrate.

Equations for components of speed of gas flow by using cylindrical coordinates could be written as

$$
\begin{gathered}
\frac{\partial v_{r}}{\partial t}=v\left[\frac{1}{r} \frac{\partial}{\partial r}\left(r \frac{\partial v_{r}}{\partial r}\right)+\frac{1}{r^{2}} \frac{\partial^{2} v_{r}}{\partial \varphi^{2}}+\frac{\partial^{2} v_{r}}{\partial z^{2}}\right]-v_{r} \frac{\partial v_{r}}{\partial r}-\frac{v_{\varphi}}{r} \frac{\partial v_{\varphi}}{\partial \varphi}-v_{z} \frac{\partial v_{z}}{\partial z}-\frac{\partial}{\partial r}\left(\frac{P}{\rho}\right) \\
\frac{\partial v_{r}}{\partial t}=v\left[\frac{1}{r} \frac{\partial}{\partial r}\left(r \frac{\partial v_{\varphi}}{\partial r}\right)+\frac{1}{r^{2}} \frac{\partial^{2} v_{\varphi}}{\partial \varphi^{2}}+\frac{\partial^{2} v_{\varphi}}{\partial z^{2}}\right]-v_{r} \frac{\partial v_{r}}{\partial r}-\frac{v_{\varphi}}{r} \frac{\partial v_{\varphi}}{\partial \varphi}-v_{z} \frac{\partial v_{z}}{\partial z}-\frac{1}{r} \frac{\partial}{\partial \varphi}\left(\frac{P}{\rho}\right) \\
\frac{\partial v_{z}}{\partial t}=v\left[\frac{1}{r} \frac{\partial}{\partial r}\left(r \frac{\partial v_{z}}{\partial r}\right)+\frac{1}{r^{2}} \frac{\partial^{2} v_{z}}{\partial \varphi^{2}}+\frac{\partial^{2} v_{z}}{\partial z^{2}}\right]-v_{r} \frac{\partial v_{r}}{\partial r}-\frac{v_{\varphi}}{r} \frac{\partial v_{\varphi}}{\partial \varphi}-v_{z} \frac{\partial v_{z}}{\partial z}-\frac{\partial}{\partial z}\left(\frac{P}{\rho}\right) .
\end{gathered}
$$

We assume, that gas in the considered reaction chamber is ideal. In this situation the following equation is correct

$$
P=\rho R T / M
$$

Equations for components of vector of speed with account the relation (6) could be written as

$$
\begin{gathered}
\frac{\partial v_{r}}{\partial t}=v\left[\frac{1}{r} \frac{\partial}{\partial r}\left(r \frac{\partial v_{r}}{\partial r}\right)+\frac{1}{r^{2}} \frac{\partial^{2} v_{r}}{\partial \varphi^{2}}+\frac{\partial^{2} v_{r}}{\partial z^{2}}\right]-v_{r} \frac{\partial v_{r}}{\partial r}-\frac{v_{\varphi}}{r} \frac{\partial v_{\varphi}}{\partial \varphi}-v_{z} \frac{\partial v_{z}}{\partial z}-\frac{R}{M} \frac{\partial T}{\partial r} \\
\frac{\partial v_{r}}{\partial t}=v\left[\frac{1}{r} \frac{\partial}{\partial r}\left(r \frac{\partial v_{\varphi}}{\partial r}\right)+\frac{1}{r^{2}} \frac{\partial^{2} v_{\varphi}}{\partial \varphi^{2}}+\frac{\partial^{2} v_{\varphi}}{\partial z^{2}}\right]-v_{r} \frac{\partial v_{r}}{\partial r}-\frac{v_{\varphi}}{r} \frac{\partial v_{\varphi}}{\partial \varphi}-v_{z} \frac{\partial v_{z}}{\partial z}-\frac{R}{r M} \frac{\partial T}{\partial \varphi}
\end{gathered}
$$


International Journal on Organic Electronics (IJOE) Vol.6, No.4, October 2017.

$$
\frac{\partial v_{z}}{\partial t}=v\left[\frac{1}{r} \frac{\partial}{\partial r}\left(r \frac{\partial v_{z}}{\partial r}\right)+\frac{1}{r^{2}} \frac{\partial^{2} v_{z}}{\partial \varphi^{2}}+\frac{\partial^{2} v_{z}}{\partial z^{2}}\right]-v_{r} \frac{\partial v_{r}}{\partial r}-\frac{v_{\varphi}}{r} \frac{\partial v_{\varphi}}{\partial \varphi}-v_{z} \frac{\partial v_{z}}{\partial z}-\frac{R}{M} \frac{\partial T}{\partial z}
$$

We calculate solution of the above system of equations by method of averaging of function corrections [7-11]. Framework the approach to determine the first-order approximations of components of flow of mixture of gases we replace the components on not yet known their average values $v v_{r} \rightarrow \alpha_{1 r}, v_{\varphi} \rightarrow \alpha_{1 \varphi}, v_{z} \rightarrow \alpha_{1 z}$ in right sides of Eqs. (5). The replacement leads to transformation of the Eqs. $(5 d)-(5 f)$ to the following form

$$
\frac{\partial v_{1 r}}{\partial t}=-\frac{R}{M} \frac{\partial T}{\partial r}, \frac{\partial v_{1 \varphi}}{\partial t}=-\frac{R}{M r} \frac{\partial T}{\partial \varphi}, \frac{\partial v_{1 z}}{\partial t}=-\frac{R}{M} \frac{\partial T}{\partial z} .
$$

Solutions of the Eqs.(7) could be written as

$$
v_{1 r}=-\frac{R}{M} \frac{\partial}{\partial r} \int_{0}^{t} T d \tau, v_{1 \varphi}=-\frac{R}{M r} \frac{\partial}{\partial \varphi} \int_{0}^{t} T d \tau, v_{1 z}=-\frac{R}{M} \frac{\partial}{\partial z} \int_{0}^{t} T d \tau .
$$

The second-order approximations of components of speed of flow of mixture of gases could be obtained by replacement of the components in right sides of Eqs. (5) on the following sums: $v_{r} \rightarrow \alpha_{2 r}+v_{1 r}, v_{\varphi} \rightarrow \alpha_{2 \varphi}+v_{1 \varphi}, v_{z} \rightarrow \alpha_{2 z}+v_{1 z}$. Equations for the second-order approximations could be written as

$$
\begin{aligned}
& \frac{\partial v_{2 r}}{\partial t}=v\left[\frac{1}{r} \frac{\partial}{\partial r}\left(r \frac{\partial v_{1 r}}{\partial r}\right)+\frac{1}{r^{2}} \frac{\partial^{2} v_{1 r}}{\partial \varphi^{2}}+\frac{\partial^{2} v_{1 r}}{\partial z^{2}}\right]-\left(\alpha_{2 r}+v_{1 r}\right) \frac{\partial v_{1 r}}{\partial r}- \\
& -\frac{\left(\alpha_{2 \varphi}+v_{1 \varphi}\right)}{r} \frac{\partial v_{1 \varphi}}{\partial \varphi}-\left(\alpha_{2 z}+v_{1 z}\right) \frac{\partial v_{1 z}}{\partial z}-\frac{R}{M} \frac{\partial T}{\partial r}(8 a) \\
& \frac{\partial v_{2 r}}{\partial t}=v\left[\frac{1}{r} \frac{\partial}{\partial r}\left(r \frac{\partial v_{1 \varphi}}{\partial r}\right)+\frac{1}{r^{2}} \frac{\partial^{2} v_{1 \varphi}}{\partial \varphi^{2}}+\frac{\partial^{2} v_{1 \varphi}}{\partial z^{2}}\right]- \\
& -\left(\alpha_{2 r}+v_{1 r}\right) \frac{\partial v_{1 r}}{\partial r}-\frac{\left(\alpha_{2 \varphi}+v_{1 \varphi}\right)}{r} \frac{\partial v_{1 \varphi}}{\partial \varphi}-\left(\alpha_{2 z}+v_{1 z}\right) \frac{\partial v_{1 z}}{\partial z}-\frac{R}{r M} \frac{\partial T}{\partial \varphi}(8 b) \\
& \frac{\partial v_{2 z}}{\partial t}=v\left[\frac{1}{r} \frac{\partial}{\partial r}\left(r \frac{\partial v_{1 z}}{\partial r}\right)+\frac{1}{r^{2}} \frac{\partial^{2} v_{1 z}}{\partial \varphi^{2}}+\frac{\partial^{2} v_{1 z}}{\partial z^{2}}\right]- \\
& -\left(\alpha_{2 r}+v_{1 r}\right) \frac{\partial v_{1 r}}{\partial r}-\frac{\left(\alpha_{2 \varphi}+v_{1 \varphi}\right)}{r} \frac{\partial v_{1 \varphi}}{\partial \varphi}-\left(\alpha_{2 z}+v_{1 z}\right) \frac{\partial v_{1 z}}{\partial z}-\frac{R}{M} \frac{\partial T}{\partial z} .(8 c)
\end{aligned}
$$

Integration of the left and time sides of Eqs. $(8 a)-(8 c)$ on time leads to the following results

$$
\begin{aligned}
v_{2 r}=v \int_{0}^{t}\left[\frac{1}{r} \frac{\partial}{\partial r}\left(r \frac{\partial v_{1 r}}{\partial r}\right)+\frac{1}{r^{2}} \frac{\partial^{2} v_{1 r}}{\partial \varphi^{2}}+\frac{\partial^{2} v_{1 r}}{\partial z^{2}}\right] d \tau-\int_{0}^{t}\left(\alpha_{2 r}+v_{1 r}\right) \frac{\partial v_{1 r}}{\partial r} d \tau- \\
\quad-\frac{1}{r} \int_{0}^{t}\left(\alpha_{2 \varphi}+v_{1 \varphi}\right) \frac{\partial v_{1 \varphi}}{\partial \varphi} d \tau-\int_{0}^{t}\left(\alpha_{2 z}+v_{1 z}\right) \frac{\partial v_{1 z}}{\partial z} d \tau-\frac{R}{M} \int_{0}^{t} \frac{\partial T}{\partial r} d \tau(8 d)
\end{aligned}
$$


International Journal on Organic Electronics (IJOE) Vol.6, No.4, October 2017.

$$
\begin{aligned}
& v_{2 r}=v \int_{0}^{t}\left[\frac{1}{r} \frac{\partial}{\partial r}\left(r \frac{\partial v_{1 \varphi}}{\partial r}\right)+\frac{1}{r^{2}} \frac{\partial^{2} v_{1 \varphi}}{\partial \varphi^{2}}+\frac{\partial^{2} v_{1 \varphi}}{\partial z^{2}}\right] d \tau-\int_{0}^{t}\left(\alpha_{2 r}+v_{1 r}\right) \frac{\partial v_{1 r}}{\partial r} d \tau- \\
&-\frac{1}{r} \int_{0}^{t}\left(\alpha_{2 \varphi}+v_{1 \varphi}\right) \frac{\partial v_{1 \varphi}}{\partial \varphi} d \tau-\int_{0}^{t}\left(\alpha_{2 z}+v_{1 z}\right) \frac{\partial v_{1 z}}{\partial z} \frac{\partial v_{1 r}}{\partial r} d \tau-\frac{R}{r} \int_{0}^{t} \frac{\partial T}{\partial \varphi} d \tau \\
& v_{2 z}=v \int_{0}^{t}\left[\frac{1}{r} \frac{\partial}{\partial r}\left(r \frac{\partial v_{1 z}}{\partial r}\right)+\frac{1}{r^{2}} \frac{\partial^{2} v_{1 z}}{\partial \varphi^{2}}+\frac{\partial^{2} v_{1 z}}{\partial z^{2}}\right] d \tau-\int_{0}^{t}\left(\alpha_{2 r}+v_{1 r}\right) \frac{\partial v_{1 r}}{\partial r} d \tau- \\
&-\frac{1}{r} \int_{0}^{t}\left(\alpha_{2 \varphi}+v_{1 \varphi}\right) \frac{\partial v_{1 \varphi}}{\partial \varphi} d \tau-\int_{0}^{t}\left(\alpha_{2 z}+v_{1 z}\right) \frac{\partial v_{1 z}}{\partial z} d \tau-\frac{R}{M} \int_{0}^{t} \frac{\partial T}{\partial z} d \tau .
\end{aligned}
$$

We determine the average values $\alpha_{2 r}, \alpha_{2 \varphi}, \alpha_{2 z}$ by the standard relations

$$
\begin{gathered}
\alpha_{2 r}=\frac{1}{\pi \Theta R^{2} L} \int_{0}^{\Theta} \int_{0}^{R} r \int_{0}^{2 \pi} \int_{-L}^{L}\left(v_{2 r}-v_{1 r}\right) d z d \varphi d r d t, \alpha_{2 \varphi}=\frac{1}{\pi \Theta R^{2} L} \int_{0}^{\Theta} \int_{0}^{R} r \int_{0}^{2 \pi} \int_{-L}^{L}\left(v_{2 \varphi}-v_{1 \varphi}\right) d z d \varphi d r d t, \\
\alpha_{2 z}=\frac{1}{\pi \Theta R^{2} L} \int_{0}^{\Theta} \int_{0}^{R} r \int_{0}^{2 \pi} \int_{-L}^{L}\left(v_{2 z}-v_{1 z}\right) d z d \varphi d r d t
\end{gathered}
$$

Here $\Theta$ is the continuance of flowing of mixture of gases. Substitution of the approximations with the first- and the second-orders into the relation (9) gives the possibility to obtain system of equations for the considered average values

$$
\left\{\begin{array}{l}
A_{1} \alpha_{2 r}+B_{1} \alpha_{2 \varphi}+C_{1} \alpha_{2 z}=D_{1} \\
A_{2} \alpha_{2 r}+B_{2} \alpha_{2 \varphi}+C_{2} \alpha_{2 z}=D_{2} \\
A_{3} \alpha_{2 r}+B_{3} \alpha_{2 \varphi}+C_{3} \alpha_{2 z}=D_{3}
\end{array}\right.
$$

Here $\quad A_{1}=1+\int_{0}^{\Theta}(\Theta-t) \int_{0}^{R} r \int_{0}^{2 \pi} \int_{-L}^{L} \frac{\partial v_{1 r}}{\partial r} d z d \varphi d r d t, \quad B_{1}=\int_{0}^{\Theta}(\Theta-t) \int_{0}^{R 2 \pi} \int_{0}^{L} \int_{-L}^{L} \frac{\partial v_{1 r}}{\partial \varphi} d z d \varphi d r d t, \quad C_{1}=C_{2}=$ $=\frac{\pi}{2} \Theta^{2} R^{2} V_{0}, D_{1}=v \int_{0}^{\Theta}(\Theta-t) \int_{0}^{R} r \int_{0}^{2 \pi} \int_{-L}^{L}\left[\frac{1}{r} \frac{\partial}{\partial r}\left(r \frac{\partial v_{1 r}}{\partial r}\right)+\frac{1}{r^{2}} \frac{\partial^{2} v_{1 r}}{\partial \varphi^{2}}+\frac{\partial^{2} v_{1 r}}{\partial z^{2}}\right] d z d \varphi d r d t-\frac{\pi}{8} \Theta^{2} \times$ $\times R^{2} V_{0}^{2}-\int_{0}^{\Theta}(\Theta-t) \int_{0}^{R} r \int_{0}^{2 \pi} \int_{-L}^{L} v_{1 r} \frac{\partial v_{1 r}}{\partial r} d z d \varphi d r d t-\int_{0}^{\Theta}(\Theta-t) \int_{0}^{R} \int_{0}^{2 \pi} \int_{-L}^{L} v_{1 \varphi} \frac{\partial v_{1 r}}{\partial \varphi} d z d \varphi d r d t, A_{2}=\int_{0}^{\Theta}(\Theta-$ $-t) \int_{0}^{R} r \int_{0}^{2 \pi} \int_{-L}^{L} \frac{\partial v_{1 r}}{\partial r} d z d \varphi d r d t, B_{2}=1+\int_{0}^{\Theta}(\Theta-t) \int_{0}^{R} \int_{0}^{2 \pi} \int_{-L}^{L} \frac{\partial v_{1 r}}{\partial \varphi} d z d \varphi d r d t, D_{2}=v \int_{0}^{\Theta}(\Theta-t) \int_{0}^{R} r \times$ $\times \int_{0}^{2 \pi} \int_{-L}^{L}\left[\frac{1}{r} \frac{\partial}{\partial r}\left(r \frac{\partial v_{1 \varphi}}{\partial r}\right)+\frac{1}{r^{2}} \frac{\partial^{2} v_{1 \varphi}}{\partial \varphi^{2}}+\frac{\partial^{2} v_{1 \varphi}}{\partial z^{2}}\right] d z d \varphi d r d t-\frac{\pi}{8} \Theta^{2} R^{2} V_{0}^{2}-\int_{0}^{\Theta}(\Theta-t) \int_{0}^{R} r \int_{0}^{2 \pi} \int_{-L}^{L} v_{1 r} \times$ $\times \frac{\partial v_{1 r}}{\partial r} d z d \varphi d r d t-\int_{0}^{\Theta}(\Theta-t) \int_{0}^{R} \int_{0}^{2 \pi} \int_{-L}^{L} v_{1 \varphi} \frac{\partial v_{1 r}}{\partial \varphi} d z d \varphi d r d t, A_{3}=\int_{0}^{\Theta}(\Theta-t) \int_{0}^{R} r \int_{0}^{2 \pi} \int_{-L}^{L} \frac{\partial v_{1 z}}{\partial r} d z d \varphi d r d t$, $B_{3}=\int_{0}^{\Theta}(\Theta-t) \int_{0}^{R} r \int_{0}^{2 \pi} \int_{-L}^{L} \frac{\partial v_{1 z}}{\partial \varphi} d z d \varphi d r d t, C_{3}=1+\frac{\pi}{2} \Theta^{2} R^{2} V_{0}, D_{3}=v \int_{0}^{\Theta} \int_{0}^{R} r \int_{0}^{2 \pi} \int_{-L}^{L}\left[\frac{1}{r} \frac{\partial}{\partial r}\left(r \frac{\partial v_{1 z}}{\partial r}\right)+\right.$ 
International Journal on Organic Electronics (IJOE) Vol.6, No.4, October 2017.

$$
\begin{aligned}
& \left.+\frac{1}{r^{2}} \frac{\partial^{2} v_{1 z}}{\partial \varphi^{2}}+\frac{\partial^{2} v_{1 z}}{\partial z^{2}}\right] d z d \varphi d r(\Theta-t) d t-\frac{\pi}{8} \Theta^{2} R^{2} V_{0}^{2}-\int_{0}^{\Theta}(\Theta-t) \int_{0}^{R} r \int_{0}^{2 \pi} \int_{-L}^{L} v_{1 r} \frac{\partial v_{1 z}}{\partial r} d z d \varphi d r d t- \\
& -\int_{0}^{\Theta}(\Theta-t) \int_{0}^{R} r \int_{0}^{2 \pi} \int_{-L}^{L} v_{1 \varphi} \frac{\partial v_{1 z}}{\partial \varphi} d z d \varphi d r d t .
\end{aligned}
$$

Solution of the system of Eqs.(10) could be determine by standard approaches [12] and could be written as

$$
\alpha_{2 r}=\Delta_{r} / \Delta, \alpha_{2 \varphi}=\Delta_{\varphi} / \Delta, \alpha_{2 z}=\Delta_{z} / \Delta
$$

where $\Delta=A_{1}\left(B_{2} C_{3}-B_{3} C_{2}\right)-B_{1}\left(A_{2} C_{3}-A_{3} C_{2}\right)+C_{1}\left(A_{2} B_{3}-A_{3} B_{2}\right), \Delta_{r}=D_{1}\left(B_{2} C_{3}-B_{3} C_{2}\right)-B_{1} \times$ $\times\left(D_{2} C_{3}-D_{3} C_{2}\right)+C_{1}\left(D_{2} B_{3}-D_{3} B_{2}\right), \Delta_{\varphi}=D_{1}\left(B_{2} C_{3}-B_{3} C_{2}\right)-B_{1}\left(D_{2} C_{3}-D_{3} C_{2}\right)+C_{1}\left(D_{2} B_{3}-\right.$ $\left.-D_{3} B_{2}\right), \Delta_{z}=A_{1}\left(B_{2} D_{3}-B_{3} D_{2}\right)-B_{1}\left(A_{2} D_{3}-A_{3} D_{2}\right)+D_{1}\left(A_{2} B_{3}-A_{3} B_{2}\right)$.

In this section we obtain the second-order approximations of components of speed of flow framework the method of averaging of function corrections. The second-order approximations is usually enough good approximations for qualitative analysis of the obtained solutions and to make several quantitative results.

Eqs.(1) and (3) with using cylindrical coordinates could be written as

$$
\begin{array}{r}
c \frac{\partial T}{\partial t}=\lambda \frac{\partial^{2} T}{\partial r^{2}}+\lambda \frac{1}{r^{2}} \frac{\partial^{2} T}{\partial \varphi^{2}}+\lambda \frac{\partial^{2} T}{\partial z^{2}}+p-c \frac{\partial}{\partial r}\left(v_{r} C T\right)-\frac{c}{r} \frac{\partial}{\partial \varphi}\left(v_{\varphi} C T\right)-c \frac{\partial}{\partial z}\left(v_{z} C T\right), \\
\frac{\partial C}{\partial t}=\frac{1}{r} \frac{\partial}{\partial r}\left(r D \frac{\partial C}{\partial r}\right)-\frac{1}{r} \frac{\partial}{\partial r}\left(r C v_{r}\right)+\frac{1}{r^{2}} \frac{\partial}{\partial \varphi}\left(D \frac{\partial C}{\partial \varphi}\right)- \\
-\frac{1}{r} \frac{\partial}{\partial \varphi}\left(C c_{\varphi}\right)+\frac{\partial}{\partial z}\left(D \frac{\partial C}{\partial z}\right)-\frac{\partial}{\partial z}\left(C c_{z}\right) .
\end{array}
$$

To calculate spatio-temporal distributions of temperature and concentration of growth component in gas mixture we also used method of averaging of function corrections. We determine the firstorder approximations by their not yet known average values $\alpha_{1 T}$ and $\alpha_{1 C}$ in right sides of the above equations. Using recently considered algorithm gives us possibility to obtain relations for the first-order approximations of the considered temperature and concentration in the following form

$$
\begin{aligned}
& T_{1}(r, \varphi, z, t)=T_{r}+\int_{0}^{t} \frac{p(r, \varphi, z, \tau)}{c} d \tau-\alpha_{1 T} \alpha_{1 C} \int_{0}^{t} \frac{\partial v_{r}(r, \varphi, z, \tau)}{\partial r} d \tau- \\
&-\frac{\alpha_{1 T} \alpha_{1 C}}{r} \int_{0}^{t} \frac{\partial v_{\varphi}(r, \varphi, z, \tau)}{\partial \varphi} d \tau-\alpha_{1 T} \alpha_{1 C} \int_{0}^{t} \frac{\partial v_{z}(r, \varphi, z, \tau)}{\partial z} d \tau, \\
& C_{1}(r, \varphi, z, t)=-\alpha_{1 T} \alpha_{1 C} \int_{0}^{t} \frac{\partial v_{r}(r, \varphi, z, \tau)}{\partial r} d \tau-\frac{\alpha_{1 C}}{r} \int_{0}^{t} \frac{\partial\left[r v_{r}(r, \varphi, z, \tau)\right]}{\partial r} d \tau- \\
&-\frac{\alpha_{1 C}}{r} \int_{0}^{t} \frac{\partial v_{\varphi}(r, \varphi, z, \tau)}{\partial \varphi} d \tau-\alpha_{1 C} \int_{0}^{t} \frac{\partial v_{z}(r, \varphi, z, \tau)}{\partial z} d \tau .
\end{aligned}
$$

We determine the average values $\alpha_{1 T}$ and $\alpha_{1 C}$ by using the standard relations 
International Journal on Organic Electronics (IJOE) Vol.6, No.4, October 2017.

$$
\begin{gathered}
\alpha_{1 T}=\frac{1}{\pi \Theta R^{2} L} \int_{0}^{\Theta} \int_{0}^{R} r \int_{0}^{2 \pi} \int_{-L}^{L} T_{1}(r, \varphi, z, \tau) d z d \varphi d r d t, \\
\alpha_{1 C}=\frac{1}{\pi \Theta R^{2} L} \int_{0}^{\Theta} \int_{0}^{R} r \int_{0}^{2 \pi} \int_{-L}^{L} C_{1}(r, \varphi, z, \tau) d z d \varphi d r d t .
\end{gathered}
$$

Substitution of the calculated the first-order approximations into the relations (16) gives a possibility to obtain the following result [12]

$$
\begin{gathered}
\alpha_{1 C}=C_{0} / L \cdot\left[1+\frac{1}{\pi \Theta R L} \int_{0}^{\Theta}(\Theta-t) \int_{0}^{2 \pi} \int_{-L}^{L} v_{r}(R, \varphi, z, t) d z d \varphi d t+\frac{\Theta V_{0}}{R L}\right], \\
\alpha_{1 T}=\left[T_{r}+\frac{1}{\pi \Theta R^{2} L} \int_{0}^{\Theta}(\Theta-t) \int_{0}^{R} r \int_{0}^{2 \pi} \int_{-L}^{L} \frac{p(r, \varphi, z, t)}{c} d z d \varphi d r d t\right]\left\{1+\frac{C_{0}}{\pi \Theta R L^{2}}\left[\int_{0}^{\Theta}(\Theta-t) \times\right.\right. \\
\left.\times \int_{0}^{2 \pi} \int_{-L}^{L} v_{r}(R, \varphi, z, \tau) d z d \varphi d t-\frac{1}{\pi \Theta R^{2}} \int_{0}^{\Theta}(\Theta-t) \int_{0}^{R} \int_{0}^{2 \pi} \int_{-L}^{L} v_{r}(r, \varphi, z, \tau) d z d \varphi d r d t+\frac{V_{0}}{2}\right] \times \\
\left.\times\left[1+\frac{1}{\pi \Theta R L} \int_{0}^{\Theta}(\Theta-t) \int_{0}^{2 \pi} \int_{-L}^{L} v_{r}(R, \varphi, z, t) d z d \varphi d t+\frac{\Theta V_{0}}{R L}\right]^{-1}\right\} .
\end{gathered}
$$

The second-order approximations of the temperature and the concentrations have been calculated framework standard procedure of method of averaging of function correction [7-11]. Framework the approach we replace the required functions in right sides of Eqs. (12) and (13) on the following sums: $T \rightarrow \alpha_{2 T}+T_{1}, C \rightarrow \alpha_{2 C}+C_{1}$. In this situation the second-order approximations could be written as

$$
\begin{gathered}
c \cdot T_{2}(r, \varphi, z, t)=\lambda \int_{0}^{t} \frac{\partial^{2} T_{1}(r, \varphi, z, \tau)}{\partial r^{2}} d \tau+\lambda \frac{1}{r^{2}} \int_{0}^{t} \frac{\partial^{2} T_{1}(r, \varphi, z, \tau)}{\partial \varphi^{2}} d \tau+\lambda \int_{0}^{t} \frac{\partial^{2} T_{1}(r, \varphi, z, \tau)}{\partial z^{2}} d \tau+ \\
+\int_{0}^{t} p(r, \varphi, z, \tau) d \tau-c \cdot \frac{\partial}{\partial r} \int_{0}^{t}\left\{v_{r}(r, \varphi, z, \tau) \cdot\left[\alpha_{2 C}+C_{1}(r, \varphi, z, \tau)\right] \cdot\left[\alpha_{2 T}+T_{1}(r, \varphi, z, \tau)\right]\right\} d \tau+ \\
+T_{r}-\frac{c}{r} \frac{\partial}{\partial \varphi_{0}^{t}} \int_{0}^{t}\left\{v_{\varphi}(r, \varphi, z, \tau) \cdot\left[\alpha_{2 C}+C_{1}(r, \varphi, z, \tau)\right] \cdot\left[\alpha_{2 T}+T_{1}(r, \varphi, z, \tau)\right]\right\} d \tau- \\
-c \cdot \frac{\partial}{\partial z} \int_{0}^{t}\left\{v_{r}(r, \varphi, z, \tau) \cdot\left[\alpha_{2 C}+C_{1}(r, \varphi, z, \tau)\right] \cdot\left[\alpha_{2 T}+T_{1}(r, \varphi, z, \tau)\right]\right\} d \tau, \\
C_{2}(r, \varphi, z, t)=\frac{1}{r} \frac{\partial}{\partial r} \int_{0}^{t} r D \frac{\partial C_{1}(r, \varphi, z, \tau)}{\partial r} d \tau+\frac{1}{r^{2}} \frac{\partial}{\partial \varphi} \int_{0}^{t} D \frac{\partial C_{1}(r, \varphi, z, \tau)}{\partial \varphi} d \tau+\frac{\partial}{\partial z} \int_{0}^{t} D \times \\
\times \frac{\partial C_{1}(r, \varphi, z, \tau)}{\partial z} d \tau-\frac{1}{r} \frac{\partial}{\partial r}\left\{r \int_{0}^{t}\left[\alpha_{2 C}+C_{1}(r, \varphi, z, \tau)\right] \cdot v_{r}(r, \varphi, z, \tau) d \tau\right\}-\frac{1}{r} \frac{\partial}{\partial \varphi_{0}^{t} v_{\varphi}(r, \varphi, z, \tau) .} \\
\cdot\left[\alpha_{2 C}+C_{1}(r, \varphi, z, \tau)\right] d \tau-\frac{\partial}{\partial z} \int_{0}^{t}\left[\alpha_{2 C}+C_{1}(r, \varphi, z, \tau)\right] \cdot v_{z}(r, \varphi, z, \tau) d \tau+C_{0} \delta(z+L) .
\end{gathered}
$$

Average values of the second-orders of the considered temperature and concentration $\alpha_{2 T}$ and $\alpha_{2 C}$ have been determined by the following standard relations 
International Journal on Organic Electronics (IJOE) Vol.6, No.4, October 2017.

$$
\alpha_{2 T}=\frac{1}{\pi \Theta R^{2} L} \int_{0}^{\Theta R} \int_{0}^{2} r \int_{0}^{2 \pi} \int_{-L}^{L}\left(T_{2}-T_{1}\right) d z d \varphi d r d t, \alpha_{2 C}=\frac{1}{\pi \Theta R^{2} L} \int_{0}^{\Theta} \int_{0}^{R} r \int_{0}^{2 \pi} \int_{-L}^{L}\left(C_{2}-C_{1}\right) d z d \varphi d r d t
$$

Substitution of the first- and the second-orders of temperature and concentration into the relations (20) gives us possibility to obtain relations for the appropriate average values in the following form

$$
\begin{aligned}
& \alpha_{2 T}=\left(\frac{\lambda \sigma}{c \pi \Theta R L} \int_{0}^{\Theta}(\Theta-t) \int_{0}^{2 \pi} \int_{-L}^{L} T^{4}(R, \varphi, z, t) d z d \varphi d t-\int_{0}^{\Theta}(\Theta-t) \int_{0}^{2 \pi} \int_{-L}^{L} T_{1}(R, \varphi, z, t) d z d \varphi d t+\right. \\
& \times \frac{\lambda}{c \pi \Theta R^{2} L}+\frac{\lambda}{c \pi \Theta R^{2} L} \int_{0}^{\Theta}(\Theta-t) \int_{0}^{2 \pi} \int_{-L}^{L} T_{1}(0, \varphi, z, t) d z d \varphi d t-\int_{0}^{\Theta}(\Theta-t) \int_{0}^{2 \pi} \int_{-L}^{L} v_{r}(R, \varphi, z, t) \times \\
& \times\left\{\left[\alpha_{2 C}+C_{1}(R, \varphi, z, t)\right] T_{1}(R, \varphi, z, t)-\alpha_{1 T} \alpha_{1 C}\right\} d z d \varphi d t \frac{1}{\pi \Theta R L}-\frac{1}{\pi \Theta R^{2} L} \int_{0}^{\Theta}(\Theta-t) \times \\
& \times \int_{0}^{R} \int_{0}^{2 \pi} \int_{-L}^{L} v_{r}(r, \varphi, z, \tau)\left\{T_{1}(r, \varphi, z, t)\left[\alpha_{2 C}+C_{1}(r, \varphi, z, t)\right]-\alpha_{1 T} \alpha_{1 C}\right\} d z d \varphi r d r d t-\frac{V_{0}}{\pi \Theta R^{2} L} \times \\
& \times \int_{0}^{\Theta}(\Theta-t) \int_{0}^{R} r \int_{0}^{2 \pi}\left[\left(\alpha_{2 C}+C_{0}\right) \cdot T_{1}(r, \varphi, L, t)-\alpha_{1 T} \alpha_{1 C}\right] d \varphi d r d t\left\{1+\frac{1}{\pi \Theta R L} \int_{0}^{\Theta 2 \pi} \int_{0}^{L} \int_{-L}^{L} v_{r}(R, \varphi, z, t) \times\right. \\
& \times\left[\alpha_{2 C}+C_{1}(R, \varphi, z, t)\right] d z d \varphi(\Theta-t) d t-\frac{1}{\pi \Theta R^{2} L} \int_{0}^{\Theta}(\Theta-t) \int_{0}^{R} r \int_{0}^{2 \pi} \int_{-L}^{L}\left[\alpha_{2 C}+C_{1}(r, \varphi, z, t)\right] \times \\
& \left.\times v_{r}(r, \varphi, z, \tau) d z d \varphi d r d t+2 V_{0}\left(\alpha_{2 C}+C_{0}\right) \frac{\Theta}{L}\right\}^{-1}, \\
& \alpha_{2 C}=\frac{1}{\pi \Theta R^{2} L} \int_{0}^{\Theta}(\Theta-t) \int_{0}^{R} r \int_{0}^{2 \pi} D\left[\left.\frac{\partial C_{1}(r, \varphi, z, \tau)}{\partial z}\right|_{z=L}-\left.\frac{\partial C_{1}(r, \varphi, z, \tau)}{\partial z}\right|_{z=-L}\right] d \varphi d r d t- \\
& -\frac{1}{\pi \Theta R^{2} L} \int_{0}^{\Theta}(\Theta-t) \int_{0}^{2 \pi} \int_{-L}^{L}\left\{r\left[\alpha_{2 C}-\alpha_{1 C}+C_{1}(R, \varphi, z, \tau)\right] \cdot v_{r}(R, \varphi, z, \tau)\right\} d z d \varphi d t- \\
& -\frac{V_{0}}{\pi \Theta R^{2} L} \int_{0}^{\Theta}(\Theta-t) \int_{0}^{R} r \int_{0}^{2 \pi}\left(\alpha_{2 C}-\alpha_{1 C}+C_{0}\right) d z d \varphi d r d t
\end{aligned}
$$

\section{DISCUSSION}

In this section we used relations $(8,9,18-20)$ analyzed spatio-temporal distributions of temperature and concentration of growth component in flow of gas mixture on frequency of rotation of disk keeper of substrate $\omega$ and diffusion coefficient during MOCVD epitaxy of gallium arsenide (see Figs. 2 and 3). The considered results have been obtained for the following case: gas-carrier hydrogen, internal diameter of reaction chamber $d=200 \mathrm{~mm}$, diameter of disk keeper of substrate is $d_{1}=160 \mathrm{~mm}$, volumetric speed of flow is 10 liter/min; frequency of rotating of keeper of substrate is $\omega=20 \mathrm{rad} / \mathrm{min}$; specific length of reaction chamber is $L=200 \mathrm{~mm}$.

Fig. $2 a$ shows monotonous increasing of the considered stationary concentration $C$ at constant value of concentration $C_{0}$ in inlet area of reaction chamber. In this situation one can obtain increasing of speed of epitaxial growth with increasing frequency of rotation $\omega$. The Fig. $2 b$ shows dependence of the considered stationary concentration on value of diffusion coefficient $D$. The figure shows, that increasing of diffusion 
International Journal on Organic Electronics (IJOE) Vol.6, No.4, October 2017.

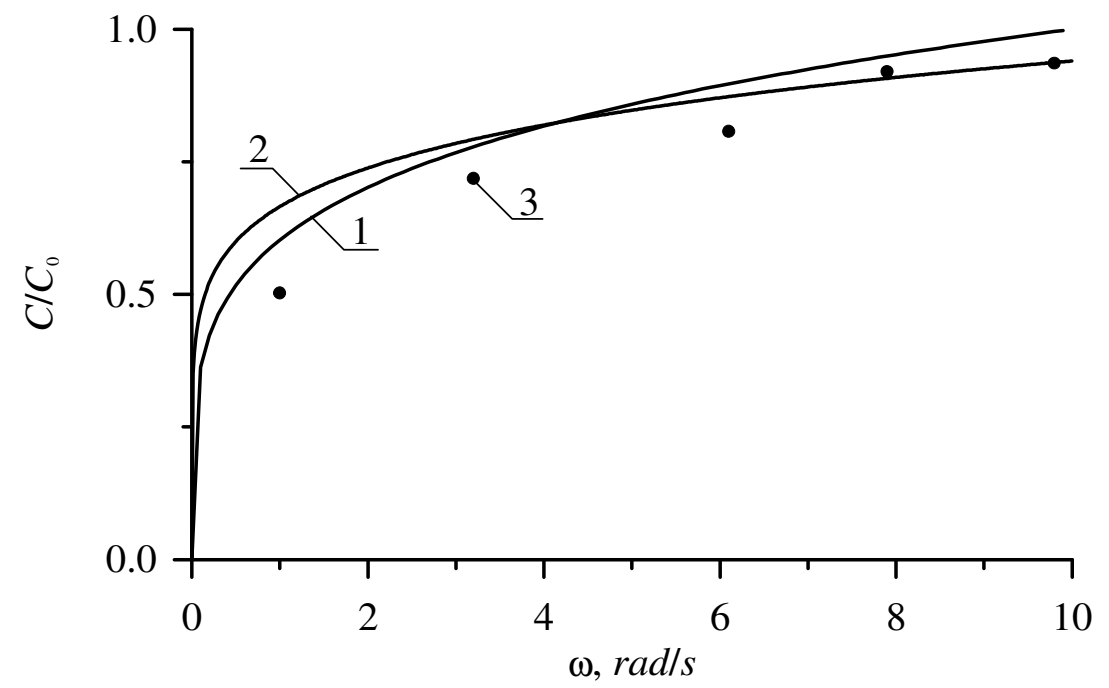

Fig. $2 a$. Dependences of stationary concentration of growth component $C$ in center of disk keeper of substrate on frequency of rotating $\omega$ at constant value of concentration $C_{0}$ in inlet area of reaction chamber. Solid lines are calculated dependences. Points are experimental results. Curve 1 corresponds to atmospheric pressure. Curve 2 corresponds to low pressure ( 0.1 of atmospheric pressure)

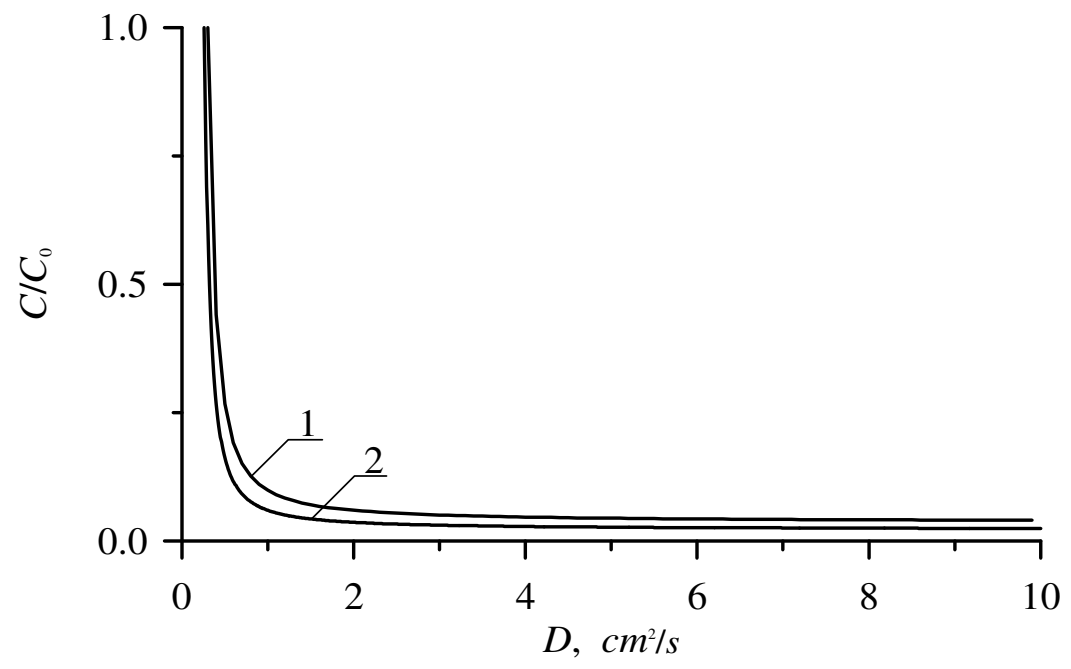

Fig. $2 b$. Dependences of stationary concentration of growth component $C$ in center of disk keeper of substrate on diffusion coefficient $D$ at constant value of concentration $C_{0}$ in inlet area of reaction chamber. Curve 1 corresponds to atmospheric pressure. Curve 2 corresponds to low pressure $(0.1$ of atmospheric pressure).

coefficient leads to increasing of speed of transport of growth component. In this situation one can find decreasing of concentration of growth component in area of growth. Curves 1 on Figs. 2 correspond to atmospheric pressure in reaction chamber. Curves 1 on Figs. 2 correspond to low pressure ( 0.1 of atmospheric pressure) in reaction chamber. Dependence of concentration of mixture of gas on kinematics viscosity $v$ is enough weak for the considered conditions of epitaxy process. In this situation we do not consider the dependence. All considered dependences (Fig. 2) show faster changing of concentration with faster changing of concentration with faster achievement of saturation with low temperature in comparison with growth of epitaxial layer at atmos- 
pheric pressure. The Fig. $2 a$ is also shows comparison of theoretical results (solid lines) with experimental one (points) for MOCVD epitaxy of gallium arsenide at low pressure. Using experimentally measured values (speed of growth of epitaxial layer and frequency of rotation of keeper of substrate) and accounting linear dependence of speed of growth on concentration of main growth component of growth component of gas mixture $C_{0}$ trimethylgallium [2,5] we obtain experimental dependence $C / C_{0}$ on $\omega$ (Fig. $2 a$ ). We find enough good coincidence of experimental dependence with experimental one. Reason of some mismatch of experimental and theoretical data is probably side reaction in gas phase [5]. Framework the reaction a part of gallium arsenide do not transit in a solid phase in reaction zone. Similar experimental dependence of $C / C_{0}$ on $\omega$ has been also obtained in [11].

Analysis of distribution of temperature near disk keeper of substrate shows more homogenous spatial distribution of the temperature in the middle of the keeper and decreasing of the temperature on the edges of the keeper because of heat losing due to convective heat exchange. Dependence of temperature in gas phase on radial coordinate $r$ is illustrated by Fig. $3 a$. We also show dependence of temperature in gas phase on axial coordinate $z$ (see Fig. 3b). The Figs. 3 show stationary distribution of temperature. Characteristics of temperature define development of reactions of individual thermal decomposition of main components and dopants of flow of mixture of gases during epitaxy of semiconductor layers.

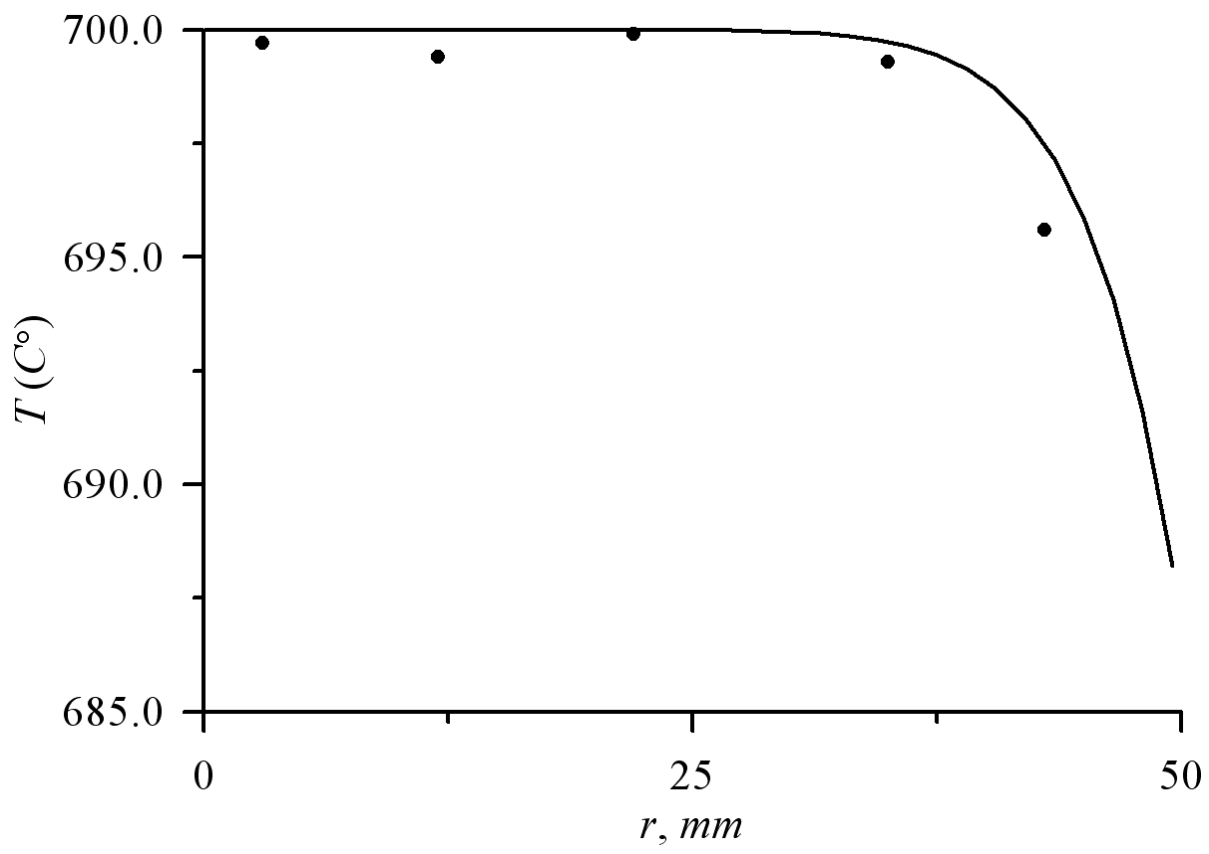

Fig. 3a. Dependences of stationary temperature in keeper of substrate on radial coordinate $z$. Points are experimental data. 
International Journal on Organic Electronics (IJOE) Vol.6, No.4, October 2017.

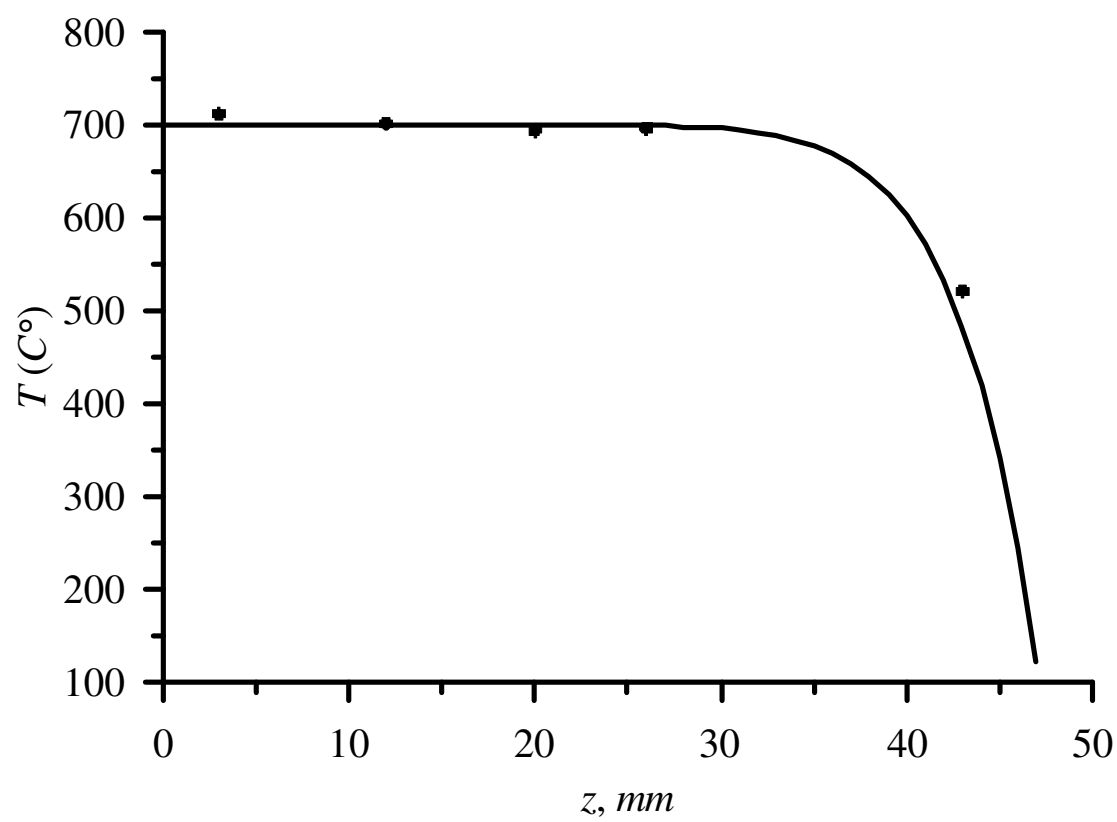

Fig. $3 b$. Dependences of stationary temperature in keeper of substrate on axial coordinate $z$. Points are experimental data.

\section{Conclusions}

In this paper we introduce an analytical approach to model of physical processes, which could be find during epitaxy from gas phase, with account rotating disk keeper of substrate. We estimate spatio-temporal distributions of concentration of growth component and temperature in flow of mixture of gases. Analysis of influence of technological parameters on the above distributions have been done. We illustrate good agreement between our calculated results and experimental one. In this situation our model is enough adequate.

\section{ACKNOWLEDGEMENTS}

This work is supported by the agreement of August 27, 2013 № 02.B.49.21.0003 between The Ministry of education and science of the Russian Federation and Lobachevsky State University of Nizhni Novgorod.

\section{REFERENCES}

[1] Molecular-Beam Epitaxy and Heterostructures. Edited L. Cheng, K.Plog, "MIR", Moscow (1989)

[2] M.A. Herman, W. Richter, H.Sitter. Epitaxy - Physical Principles and Technical Implementation, Springer, Berlin (2004)

[3] G.B. Stringfellow Organometallic Vapor-Phase Epitaxy: Theory and Practice. Academic Press, Boston (1989)

[4] R.A. Talalaev, E.V. Yakovleva, S.Yu. Karpova, Yu.N. Makarov. on Low Temperature Kinetic Effects in Metal-Organic Vapor Phase Epitaxy of III-V Compounds J.Cryst. Growth Vol. 230. P. 232-238 (2001) 
[5] W.V.Lundin, A.V. Sakharov, E.E. Zavarin, M.A. Sinitsyn, A.E. Nikolaev, G.A. Mikhailovsky, P.N. Brunkov, V.V. Goncharov, B.Ya. Ber, D.Yu Kazantsev, A.F. Tsatsul'Nikov. Effect of Carrier Gas and Doping Profile on the Surface Morphology of MOVPE Growth Heavily Doped Gan:Mg Layers. Semoconductors, Vol. 43 (7). P. 963-967 (2009).

[6] H.S. Carslaw, J.C.Jaeger. Conduction of Heat in Solids. Oxford: At the Clarendon Press 1964. 488 P

[7] Yu. D. Sokolov. About the Definition of Dynamic Forces in the Mine Lifting. Applied Mechanics. Applied Mechanics. Vol 1 (1). P. 23-35 (1955)

[8] E.L. Pankratov. Decreasing of Depth of P-N-Junction in a Semiconductor Heterostructure by Serial Radiation Processing and Microwave Annealing. J. Comp. Theor. Nanoscience. Vol 9 (1). P. 41-49 (2012).

[9] E.L.Pankratov, E.A. Bulaeva. Decreasing of Quantity of Radiation Defects in an Implanted-Junction Rectifiers by Using Overlayers. Internatioanl Journal of Micro-Nano Scale Transport. Vol 3 (3). P. 119-130 (2012).

[10] E.L. Pankratov, E.A.Bulaeva. Doping of Materials During Manufacture P-N-Junctions and Bipolar Transistors. Analytical Approaches to Model Technological Approaches and Ways of Optimization of Distributions of Dopants. Reviews in Theoretical Science Vol. 1 (1). P. 58-82 (2013).

[11] E.L. Pankratov, E.A. Bulaeva On Optimization of Regimes of Epitaxy from Gas Phase. Some Analytical Approaches to Model Physical Processes in Reactors for Epitaxy from Gas Phase During Growth Films. Reviews in Theoretical Science. Vol 3 (4). P. 365-398 (2015).

[12] G. Korn, T. Korn. Mathematical Handbook for Scientists and Engineers. Definitions, Theorems and Formulas for Reference and Review. Second Edition. McGraw-Hill Book Company New York, 1968

\section{AUTHORS}

Pankratov Evgeny Leonidovich educated in Radiophysical department of Nizhny Novgorod State University (Doctor of Science). He has 190 published papers in area of his researches.

Bulaeva Elena Alexeevna was educated in Engineering and construction department of Nizhny Novgorod State University of Architecture and Civil Engineering (Master of Science). She has 130 published papers in area of her researches. 